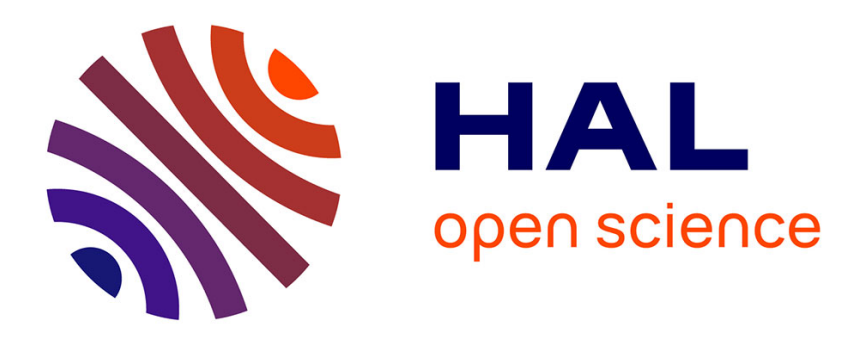

\title{
Chalcogenide-based Ultra-uniform Narrow Bandpass Filters
}

\author{
A Bourgade, F Lemarquis, A Moreau, T Begou, Julien Lumeau
}

\section{To cite this version:}

A Bourgade, F Lemarquis, A Moreau, T Begou, Julien Lumeau. Chalcogenide-based Ultra-uniform Narrow Bandpass Filters. SVC Bulletin, 2020. hal-02996122

\section{HAL Id: hal-02996122 \\ https://hal-amu.archives-ouvertes.fr/hal-02996122}

Submitted on 9 Nov 2020

HAL is a multi-disciplinary open access archive for the deposit and dissemination of scientific research documents, whether they are published or not. The documents may come from teaching and research institutions in France or abroad, or from public or private research centers.
L'archive ouverte pluridisciplinaire HAL, est destinée au dépôt et à la diffusion de documents scientifiques de niveau recherche, publiés ou non, émanant des établissements d'enseignement et de recherche français ou étrangers, des laboratoires publics ou privés. 


\section{A PUBLIGATION FOR THE VAGUUM GOATING INDUSTRY SUMMER 2020}

\section{SVC \\ |||||||||||||||| \\ SOCIETYof \\ VACUUM \\ COATERS}
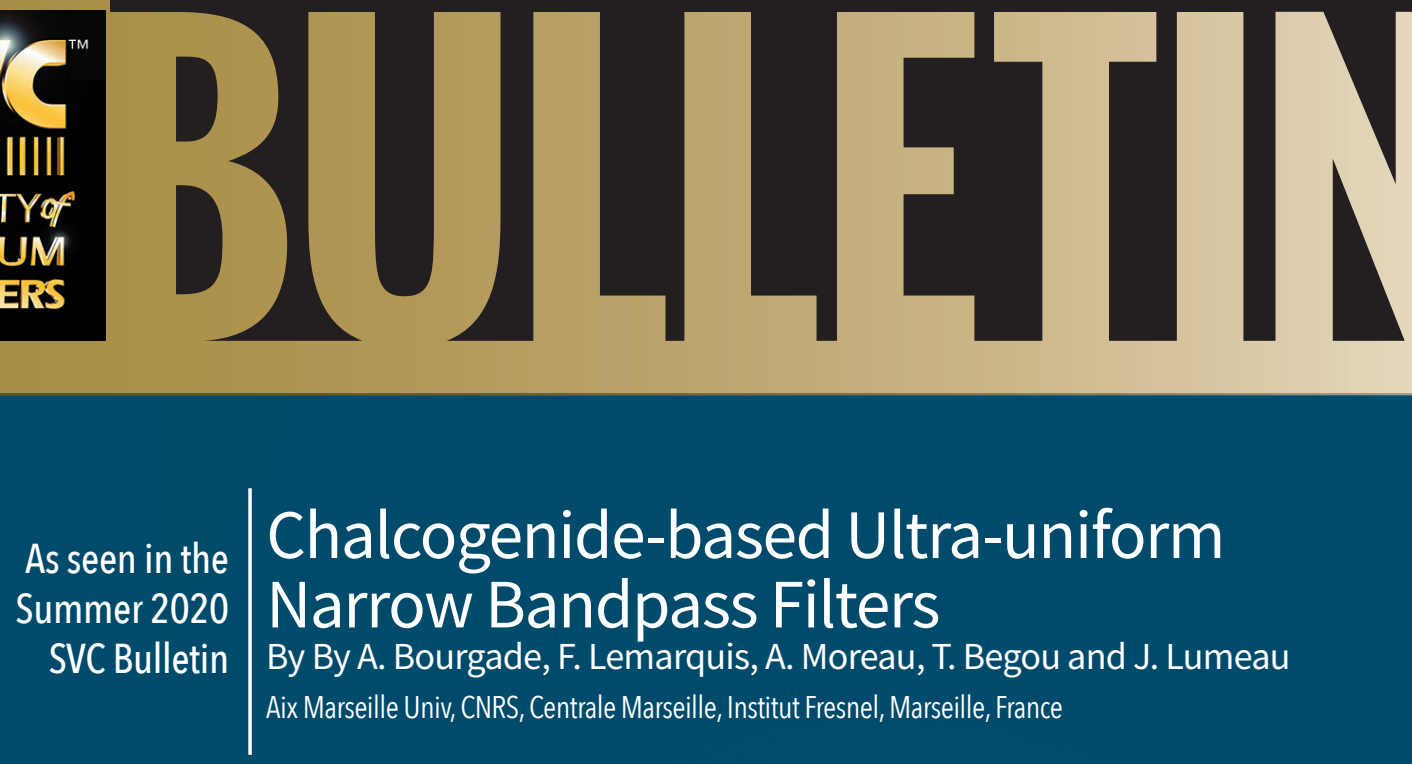

$64^{\text {th }}$ SVC Technical Conference

Symposium on

Thin Film Solutions

for a Sustainable Future

May 1 - May 6, 2021 | Nashville, TN

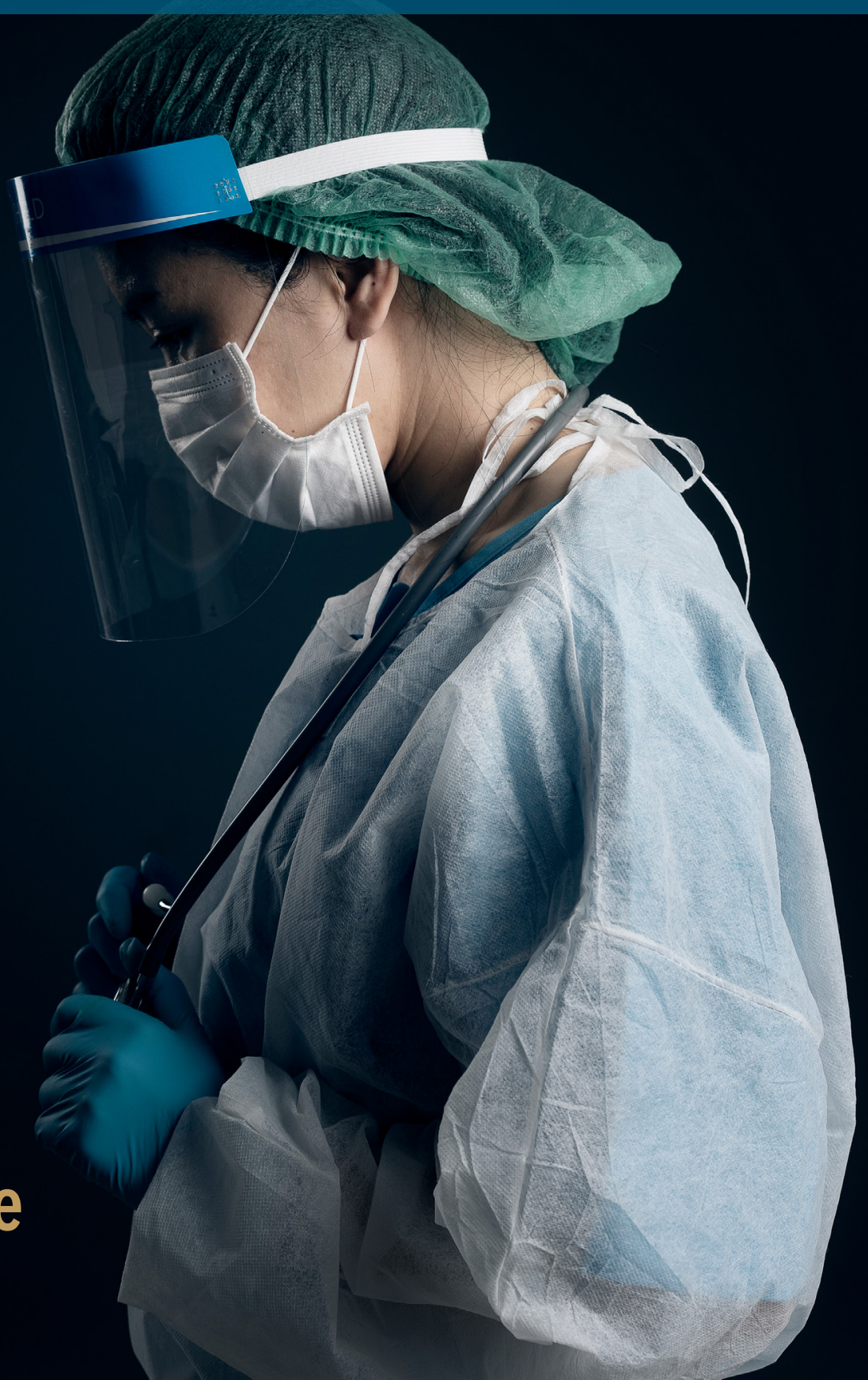




\section{CONTRIBUTED ORIGINALARTICLE}

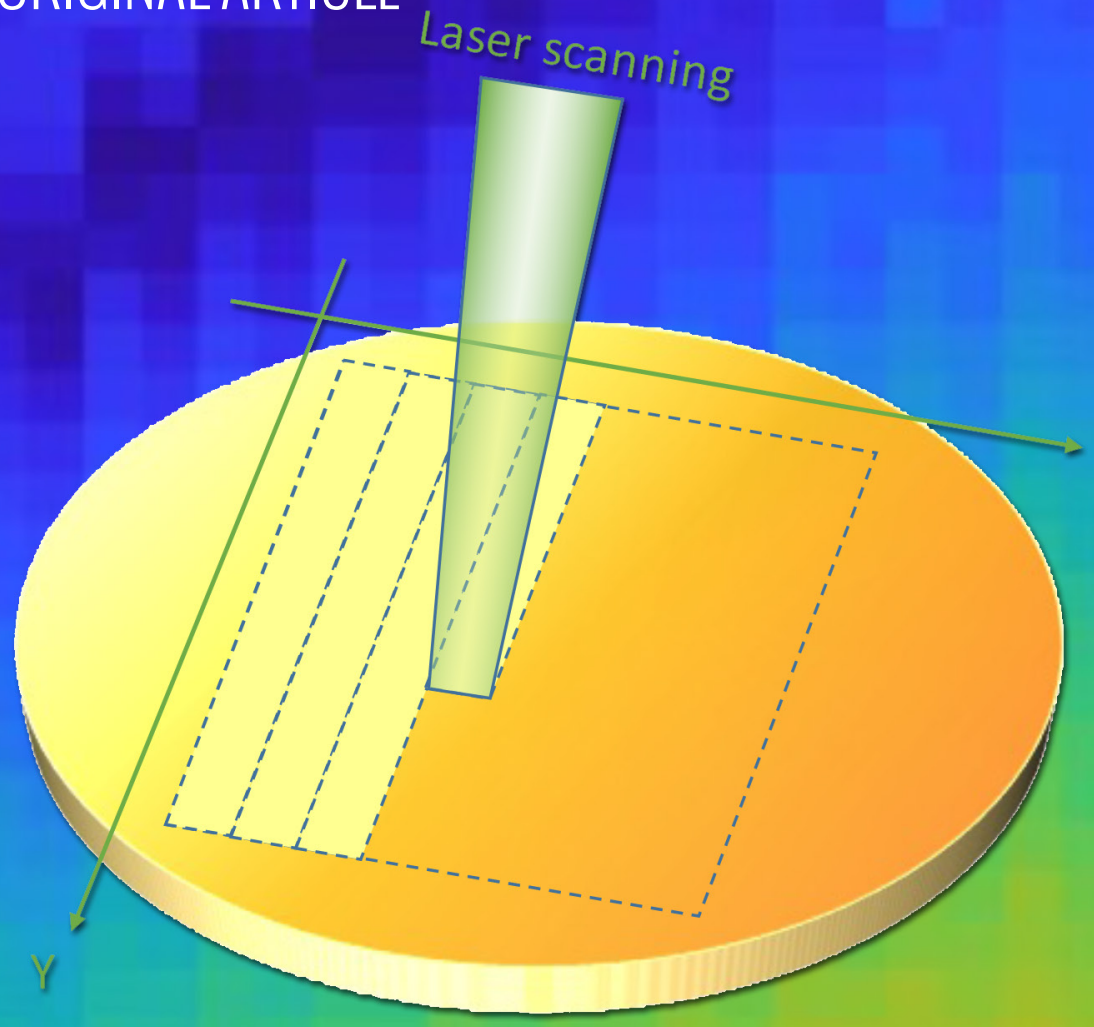

\section{Chalcogenide-based} Ultra-uniform Narrow Bandpass Filters
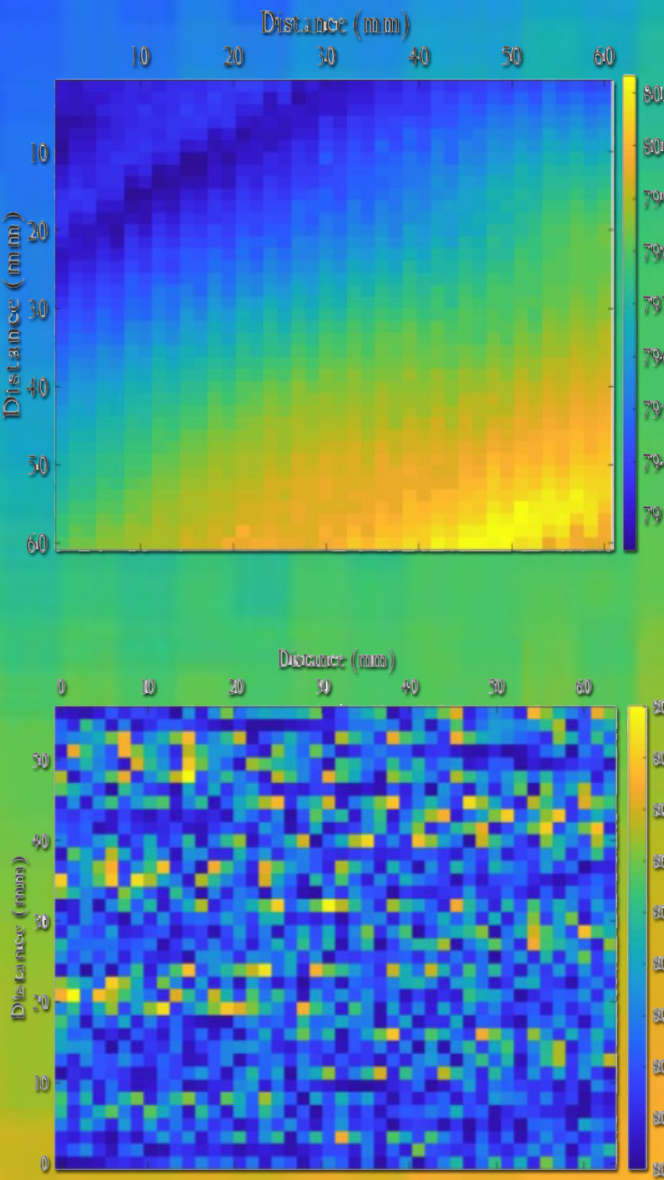

By A. Bourgade, F. Lemarquis, A. Moreau,

T. Begou and J. Lumeau

Aix Marseille Univ, CNRS, Centrale Marseille, Institut Fresnel, Marseille, France

1

Very large progress has been made within the fabrication method and the complexity of optical thin film filters. Coatings can now be accurate at nanometric scales even for stacks up to hundreds of layers. However, more and more uniform and performant filters are now needed. After filter fabrication the spectral performances of a filter are fixed. To overcome this limitation, materials which refractive index or thickness can be locally changed after exposure with an actinic radiation can be used.

In this paper, we present the fabrication of bandpass filters based on photosensitive optical thin films $\left(\mathrm{As}_{2} \mathrm{~S}_{3}\right)$. Filters centered at $800 \mathrm{~nm}$, with bandwidth of $1 \%$ or below and rejecting the whole silicon detector sensitivity spectral range are demonstrated. These filters are composed with single or multiple Fab-
ry-Perot cavities and have a nominal uniformity of $1 \%$ over 100 $\mathrm{mm}$ diameter aperture. By using the photosensitive properties of the chalcogenide layers, we show that filters with uniformity below $\pm 0.05 \%$ can be achieved. The fabricated filters are shown to have identical performances when used locally or full aperture. 


\section{INTRODUCTION}

For the past couple of decades, there has been very large progress within the fabrication method and the complexity of optical thin film filters. Coatings can now be accurate at nanometric scales even for stacks up to hundreds of layers. However, more and more uniform and performant filters are now needed, requiring better and better control of the processes during the deposition. Spectral performances of a filter are related to the optical thickness of each layer and performances are fixed at filter achievement. Moreover, due to variations of the deposited thickness over the substrate aperture, the performances of the filters vary accordingly. This problem is particularly important in case of narrow bandpass filters. Actually, it can be shown [1] that the relative variation of the central wavelength $(\lambda)$ of a bandpass filter is directly related to the relative variation of the thickness (nt) of its cavity:

$$
\frac{\Delta \lambda}{\lambda}=\kappa \frac{\Delta n t}{n t}
$$

Where $\mathrm{k}$ is a factor taking into account the phase dispersion derivative of the mirrors that we considered as equal to one in this paper. As of today, the best deposition techniques allow securing a close to $0.1 \%$ change of the thickness over a $100 \mathrm{~mm}$ aperture [2]. This non uniformity results in variation of the central wavelength of $1 \mathrm{~nm}$ for a filter centered at $1 \mu \mathrm{m}$. But most of the standard deposition techniques based on evaporation by sputtering are generally resulting in uniformities within the 1\%-range such as it is not possible to fabricate large aperture filters with bandwidth smaller than $10 \mathrm{~nm}$.

In this paper, we propose to overcome this limitation by using materials which optical thickness can be changed after deposition. The concept of such an approach was introduced some time ago [3] and basic proof-of-concept was published about 10 years ago [4]. This approach relies on the fabrication of bandpass filters made of photosensitive materials in order to locally correct the central wavelength of the filter using photo-induced refractive index change. However, in ref. [4], only small $5 \times 5 \mathrm{~mm}^{2}$ uniform bandpass filter was demonstrated. In this paper, we present a thorough analysis of the fabrication of large aperture narrowband pass filters at $800 \mathrm{~nm}$. We first present a characterization of the photosensitivity of thin $\mathrm{As}_{2} \mathrm{~S}_{3}$ films and then the use of this material for the fabrication of high performance bandpass Fabry-Perot filter. We then present a method for automatic local correction of a bandpass filter and demonstrate a highly uniform $60 \times 60 \mathrm{~mm}^{2}$-square filter with $5 \mathrm{~nm}$ bandpass and $\pm 0.1 \%$ fluctuations of the central wavelength [5].

\section{SINGLE CAVITY BANDPASS FILTERS}

For the development and manufacturing of photosensitive optical thin films, we used chalcogenide glasses such as $\mathrm{As}_{2} \mathrm{~S}_{3}$ also known as AMTIR-6. This glass is known for its broad transparency in the near and far infrared (AMTIR stands for Amorphous Material Transmitting Infrared Radiations) and has the unique characteristic of being photosensitive, i.e. some of its opto-geometrical properties can be modified after exposure to actinic radiation. This characteristic has already been demonstrated in linear regime $[6,7]$ and non-linear one (two photons absorption) [8]. In previous research, it has been shown that the photo-induced refractive index change can go up to 0.016 [9]. Many applications have also been demonstrated with such a material including waveguides for example $[6,10]$. This material and its photosensitivity has also been used to create gratings and lenslets on the surface of those films with above band gap illumination $[11,12]$. But, to the best of our knowledge, no optical quality coatings compatible with the manufacturing of complex optical elements have ever been fabricated. The chalcogenide layers and thin film stacks were produced using electron beam deposition in a Bühler SYRUSpro710 machine. The whole deposition was monitored by optical monitoring using a Bühler OMS 5000 system and deposition rate was controlled with quartz-crystal monitoring system. The material used to deposit is a bulk amorphous $\mathrm{As}_{2} \mathrm{~S}_{3}$ glass blank. The residual pressure inside the vacuum chamber was around $\sim 10^{-6} \mathrm{mbar}$, and the deposition rate was set to $3 \AA / \mathrm{s}$. The chalcogenide layers were fabricated using electron beam deposition while the Bragg mirrors of the Fabry Perot Filter (made with alternated $\mathrm{Nb}_{2} \mathrm{O}_{5}$ and $\mathrm{SiO}_{2}$ layers) were fabricated using plasma assisted electron beam deposition. Due to the photosensitive nature of such a material, we protected the samples from any ambient light exposure by keeping them in dark boxes, except during the exposure process.

To characterize the photosensitive properties of the $\mathrm{As}_{2} \mathrm{~S}_{3}$ layers, we deposited $990 \mathrm{~nm}$ thick ( $3 \lambda / 2$ at $1550 \mathrm{~nm}$ ) single layers. Then, using a Perkin Elmer Lambda 1050 spectrophotometer, we measured the spectral dependence of the transmission before and after exposing the layer to a $20 \mathrm{~mW}$ LED at $470 \mathrm{~nm}$ (Fig. 1). One can see that after exposing the layer with a dosage of 110 $\mathrm{J} / \mathrm{cm}^{2}$, the transmission spectrum is shifted to longer wavelengths by $3.5 \%$ and the amplitude of the intensity modulation is also increased by $6.4 \%$. Reverse engineering on these two curves [13] allows to show that at $800 \mathrm{~nm}$, the refractive index is changed from 2.412 to 2.497 without any change of the layer thickness.

To better characterize the refractive index change kinetics, we then exposed the $\mathrm{As}_{2} \mathrm{~S}_{3}$ layer with different dosages, measured the spectral dependence of the transmission and reflection after each dosage increment and finally implemented a reverse engineering technique to extract the opto-geometrical parameters of the layer. Fig. 2 shows the evolution of the refractive index change in $\mathrm{As}_{2} \mathrm{~S}_{3}$ layer for exposure dosage from 0 to $110 \mathrm{~J} / \mathrm{cm}^{2}$. A refractive index increment as large as 0.08 at $800 \mathrm{~nm}$ can be induced with a dosage of $\sim 20 \mathrm{~J} / \mathrm{cm}^{2}$. For larger dosages, a saturation of the refractive index is observed. In addition, it is worth noting that no change of the layer thickness could be detected 


\section{ORIGINAL ARTICLE CHALCOGENIDE-BASED ULTRA-UNIFORM NARROW BANDPASS FILTERS}

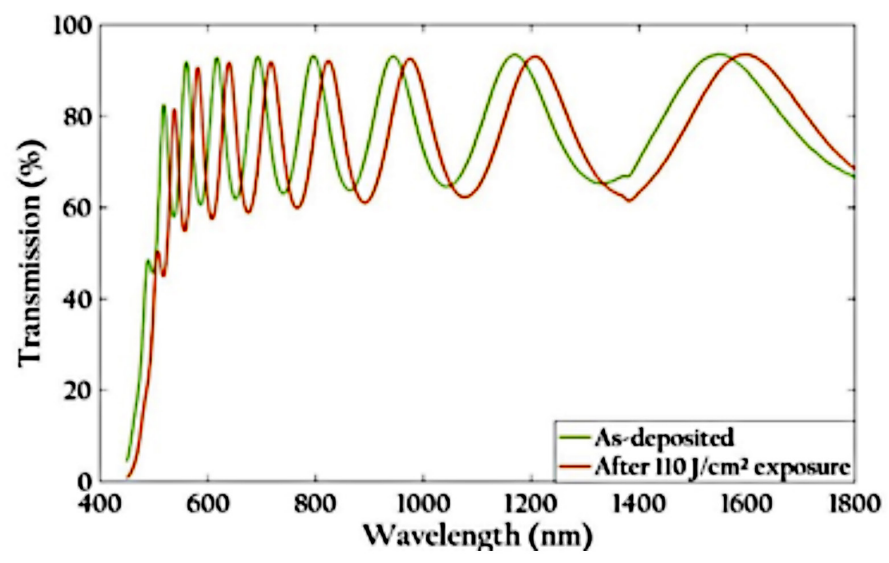

Fig. 1 - Evolution of the spectral dependence of the transmission of a $990 \mathrm{~nm}$ thick $\mathrm{As}_{2} \mathrm{~S}_{3}$ layer (green: as deposited layer and red: layer after exposure to a $20 \mathrm{~mW}$ LED at $470 \mathrm{~nm}$ ).

with our reverse engineering method and this was confirmed by optical profilometer measurements. Finally, the observed refractive index changes are non-reversible and stable (at least for more than 12 months - time since we started this work) at room temperature.

We then analyzed how the $\mathrm{As}_{2} \mathrm{~S}_{3}$ layer can be integrated within a multilayer structure. We designed a narrow bandpass filter centered at $800 \mathrm{~nm}$ with a single cavity Fabry-Perot structure, i.e. composed of one $\mathrm{As}_{2} \mathrm{~S}_{3}$ cavity separated by two dielectric quarter wave layer-based mirrors. The materials used for the mirrors are silica $\left(\mathrm{SiO}_{2}\right)$ as low index material and niobia $\left(\mathrm{Nb}_{2} \mathrm{O}_{5}\right)$ as high refractive index material. The design of the filter is:

$$
(\mathrm{HL})^{4} / 4 \mathrm{ChG} /(\mathrm{LH})^{4}
$$

where $\mathrm{H}$ stands for a quaterwave $\mathrm{Nb}_{2} \mathrm{O}_{5}$ layer, $\mathrm{L}$ for a quarterwave $\mathrm{SiO}_{2}$ layer and $\mathrm{ChG}$ for a quarterwave $\mathrm{As}_{2} \mathrm{~S}_{3}$ layer. Such a filter generates a narrow bandpass with a Full Width at Half Maximum (FWHM) of $4 \mathrm{~nm}$. A prototype of this filter was fabricated within our technological platform on a 4-inch diameter glass substrate.

After deposition, the filter was characterized with a Perkin Elmer Lambda 1050 spectrophotometer between 450 and 1100 nm. In Fig. 3, we overlapped the measured (blue curve) and theoretical (red curve) spectral dependence of the transmission of the filter. One can see that the overall experimental transmission curve is very similar to the theoretically calculated curve. However, one can see that the central wavelength is blue shifted while the side of the mirrors are red-shifted. This observation shows that the cavity was not perfectly matched during the fabrication. In addition, as the optical monitoring was done on a separate test glass, this error might also be due to inherent non-uniformity of the deposition. Indeed, it is well known that the thickness of the deposited layers will vary over the filter aperture resulting in a variation of the spectral performance. Such effect becomes noticeable and non-negligible with 4-inch diameter filters. In order to characterize this uniformity, we developed a dedicated optical setup (Fig. 4).

It is composed with a collimated white-light source. The $1 \mathrm{~mm}$ square beam is sent to the filter to be characterized and

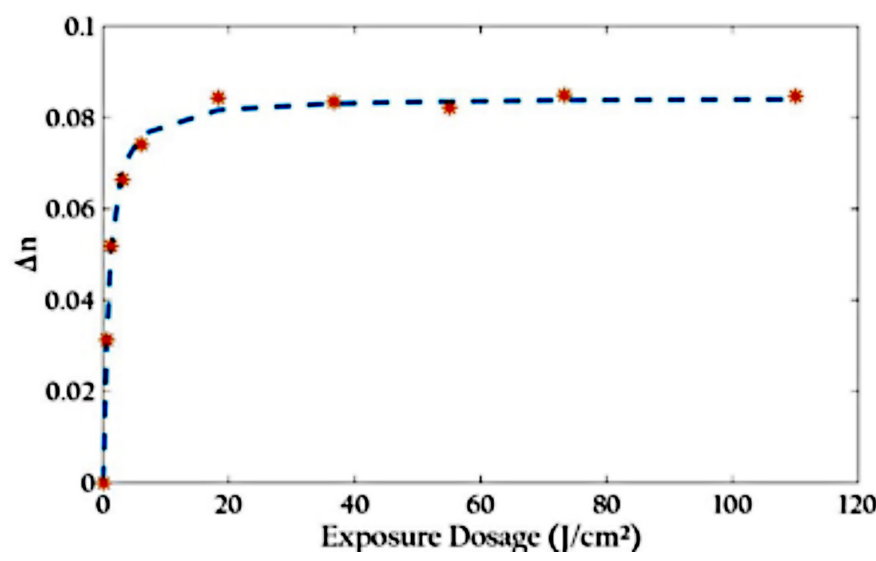

Fig. 2 - Evolution of the refractive index change as a function of the exposure dosage at $470 \mathrm{~nm}$.

the transmitted beam is then collected within a second fiber, sent to a Wasatch Photonics VIS-NIR spectrometer and analyzed within the [400-1100] nm spectral range with a $0.6 \mathrm{~nm}$ spectral resolution. The sample is placed on two translation stages in order to scan the local transmission of the filter.

The local spectral dependence of the transmission was measured over $18001 \mathrm{~mm}^{2}$ regions on a $65 \times 60 \mathrm{~mm}^{2}$ aperture. We then plotted in Fig. 5 an overlay of the measured transmission curves within the bandpass region. One can see that the filter is centered around $800 \mathrm{~nm}$ but the central wavelength shows some variations up to $9.2 \mathrm{~nm}$, i.e. $1.1 \%$ variation of the central wavelength over the filter aperture. This dispersion illustrates the non-uniformity of the SYRUSpro machine and is within the expected performances with such a deposition system. Such a dispersion of the central wavelength makes this filter not usable over its whole aperture as the summing of the spectral perfor-

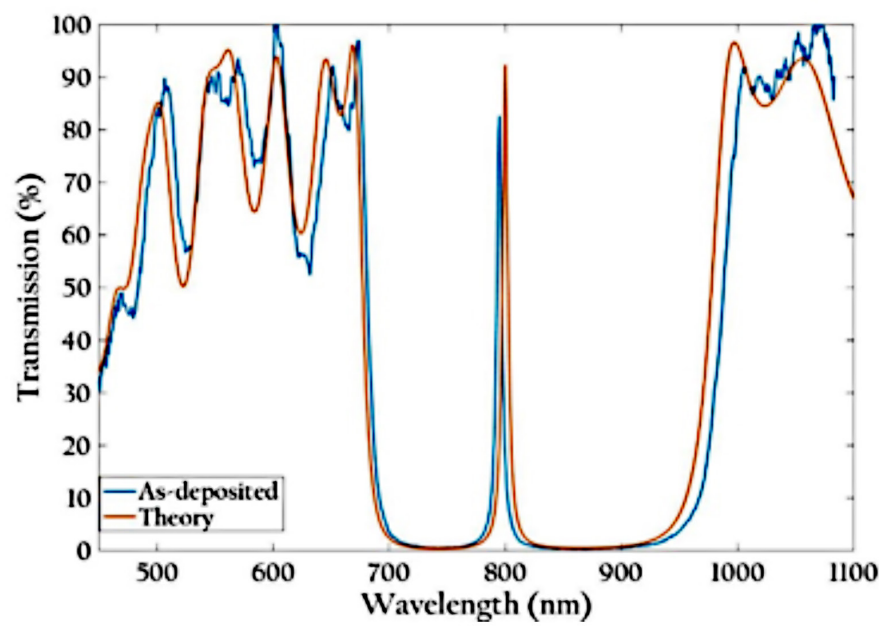

Fig. 3 - Spectral dependence of the transmission of a $(\mathrm{HL})^{4} / 4 \mathrm{ChG} /$ $(\mathrm{LH})^{4}$ Fabry Perot filter. Theoretical filter: red curve, experimental filter: blue curve. 


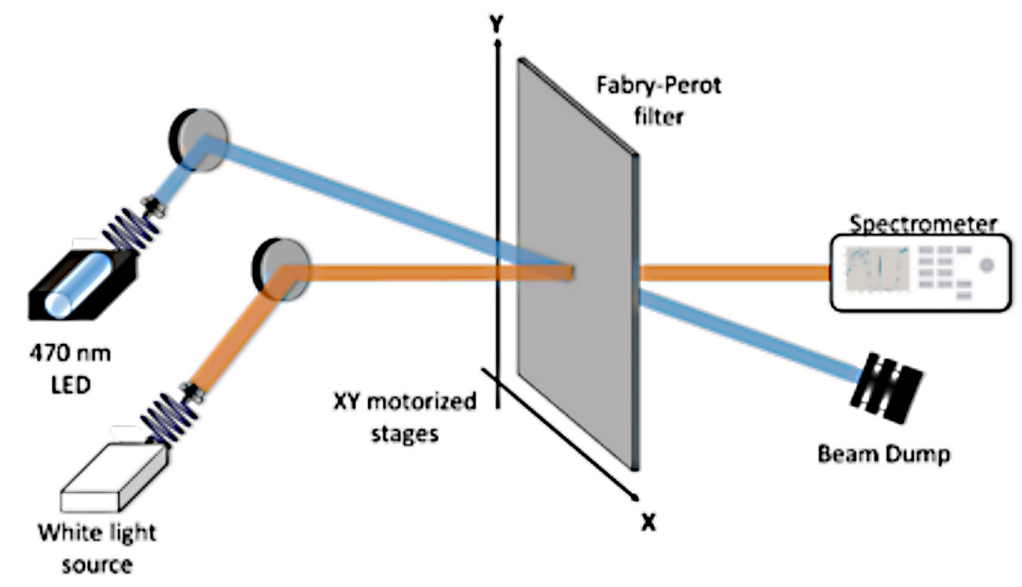

Fig. 4 - Scheme of the pump/probe experimental setup used to both characterize the local spectral performances in transmission of the filter and correct the local central wavelength of the filter. mance over its aperture will result in deteriorated spectral performances. In this work, we implemented a similar approach to that was presented by Shen et al. [13]. However, in order to demonstrate that this technique is reliable and predictable, we developed a pump/probe system that allowed automatic correction of the filter for each of the 1800 measured points (Fig. 4). Indeed, we added to the spectrophotometric system (probe system) an exposure system (pump system) composed with a blue LED of $20 \mathrm{~mW}$ emitting at $470 \mathrm{~nm}$. This exposure system produces a $1 \mathrm{~mm}$ square spot that perfectly overlaps the transmission measurement spot.

Then, at first, we characterized the kinetics of central wavelength change versus exposure dosage on a single point. Dosage was increased up to $110 \mathrm{~J} / \mathrm{cm}^{2}$ with a $5 \mathrm{~J} / \mathrm{cm}^{2}$ step and the transmission spectrum was measured after each of these steps. By increasing the dosage, the central wavelength of the filter is red-shifted but no change of the transmission could be detected (within the precision of the measurement $( \pm 1 \%)$, confirming that the refractive index change does not induce losses within the layer. We plotted in Fig. 6 the evolution of the central wavelength as a function of the dosage. We can clearly see that the evolution of the central wavelength is similar to that of refractive index change that was measured in $\mathrm{As}_{2} \mathrm{~S}_{3}$ single layers. The kinetics are however different (4 times decrease) as the electric field distribution within the cavity at $470 \mathrm{~nm}$ induces a decrease of the local effective exposure dosage. A maximum change of the central wavelength of $+10 \mathrm{~nm}$, i.e. $1.2 \%$ can be induced with this technique. This value is therefore large enough to be able to locally correct each of the measured spectrum and make the filter uniform.

To achieve very uniform filter, we decided to center the whole filter at $801 \mathrm{~nm}$ with a precision of $0.1 \%$, i.e. 10 times better than the original one. In this case, only red-shift of the filter is necessary. This case is not a limited case since if a more specific wavelength would be needed, it could be possible to adjust the fabrication procedure to guaranty that the part of the filter that has the largest central wavelength is below that targeted one.

We developed a LabVIEW program that allows to sequentially measure the local transmission, expose the sample at $470 \mathrm{~nm}$ and then measure the transmission again and repeating this procedure until the value of $801 \pm 0.8 \mathrm{~nm}$ (i.e. compatible with

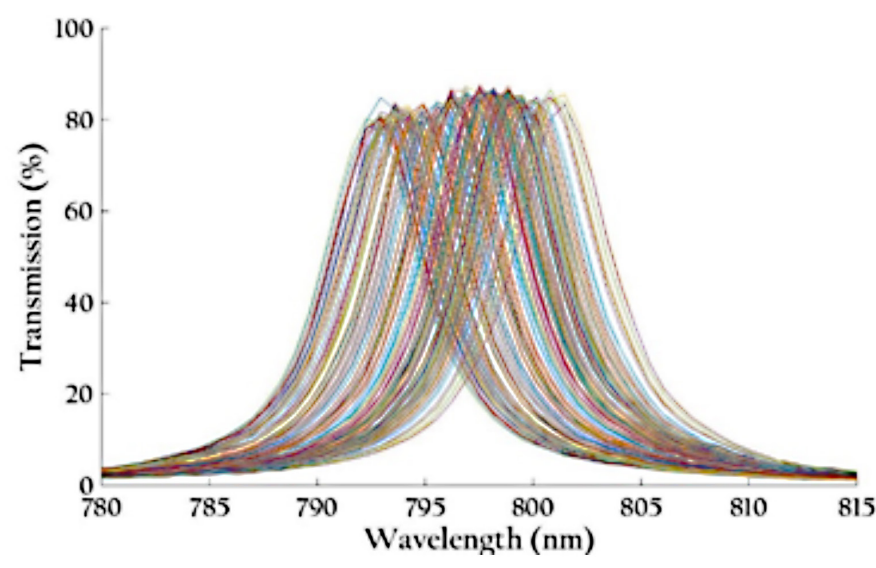

Fig. 5 - Evolution of the spectral dependence of the transmission measured on a Fabry-Perot filter. Each curve was measured on a $1 \mathrm{~mm}^{2}$ spot size over a $65 \times 60 \mathrm{~mm}^{2}$ aperture.

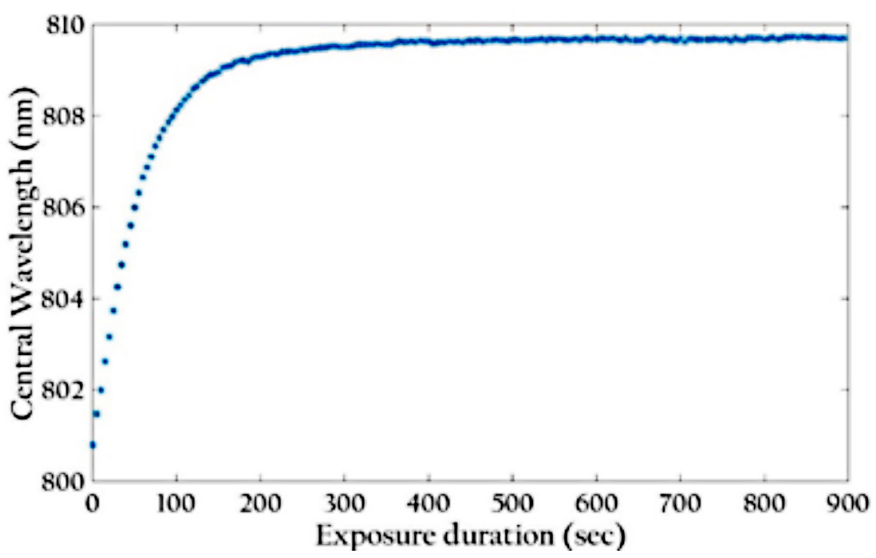

Fig. 6 - Dependence on dosage of the central wavelength peak. 


\section{ORIGINAL ARTICLE CHALCOGENIDE-BASED ULTRA-UNIFORM NARROW BANDPASS FILTERS}

the maximum measured central wavelength on the filter) is achieved. This procedure is then repeated for each of the 1800 points.

After this point-by-point correction of the local transmission of the filter, we plotted in Fig. 7 the evolution of the central wavelength of the filter over its aperture as well as an overlay of the measured transmission spectra after correction. The variations of the central wavelength of the filter over its aperture are now 10 times lower (going from $800.8 \mathrm{~nm}$ to $801.7 \mathrm{~nm}$ ) than before correction. Using this technique, the central is controlled with a $\pm 0.05 \%$ precision range over a $65 \times 60 \mathrm{~mm}^{2}$ aperture. This value is equal, if not better than what can be achieved with highly uniform deposition machines [2].

In order to confirm that the filter can now be used in full aperture, we modified the set-up in Fig. 4 and expanded the illuminating beam to a diameter of $50 \mathrm{~mm}$. The collecting collimator was also adapted in order to be able to collect, in the spectrometer, the whole transmitted beam and therefore be able to characterize the average transmission spectrum. This measurement was carried out before and after correction of the filter and was compared to the theoretical transmission of the filter (Fig. 8). Theoretically, the filter is expected to have a FWHM of 4 $\mathrm{nm}$ and a maximum transmission of $92 \%$. The as-deposited filter presents a FWHM of $\sim 10 \mathrm{~nm}$ and a maximum of transmission of $\sim 50 \%$. These deteriorated performances are due to the fact that with a large beam, the measured transmittance is the average of the transmission spectra over the filter aperture. Due to the non-uniformity which is $2.5 \times$ the FWHM, both the FHWM and the transmittance are highly affected. After correction, the variation of the central wavelength has been decreased by 10 times and is now only $0.25 \times$ the FWHM. The transmission of the filter becomes much closer to the theoretical one, i.e. we have the FWHM $=5 \mathrm{~nm}$ and the maximum transmittance $=85 \%$ ).

\section{TWO-CAVITY BANDPASS FILTERS}

Most of the applications that require the use of bandpass filters generally require filters with higher performances, i.e. with a spectral profile in the bandpass which is more square and with higher rejection close to the bandpass. This can be achieved by combining several Fabry-Perot cavities in a coherent way. In order to secure that the two cavities will shift simultaneously when exposed to laser radiation, we modified the design and opted for non-quarter wave layer structures. A detailed of the design approach can be found in ref. [15]. The structure of the filter is:

$$
\text { M6/1.85C/M6/L/M6/1.85C/M6/AR }
$$

Where $\mathrm{M} 6$ are non-quarter mirrors and $\mathrm{C}$ are the chalcogenide cavity layers. Such a filter was fabricated with the Bühler SYRUSpro 710 deposition machine and the spectral performances of the filters were the characterized using a Perkin Elmer Lambda 1050 spectrophotometer. Fig. 9 shows the broadband spectral performances of the filter. Good agreement between experimental and theoretical curves can be observed.

This filter was then exposed with a green laser at $532 \mathrm{~nm}$ and the spectral performances around the bandpass region were measured (Fig. 10). One can see that the central bandpass can be shifted towards longer wavelength by $\sim 1.2 \%$ without significant
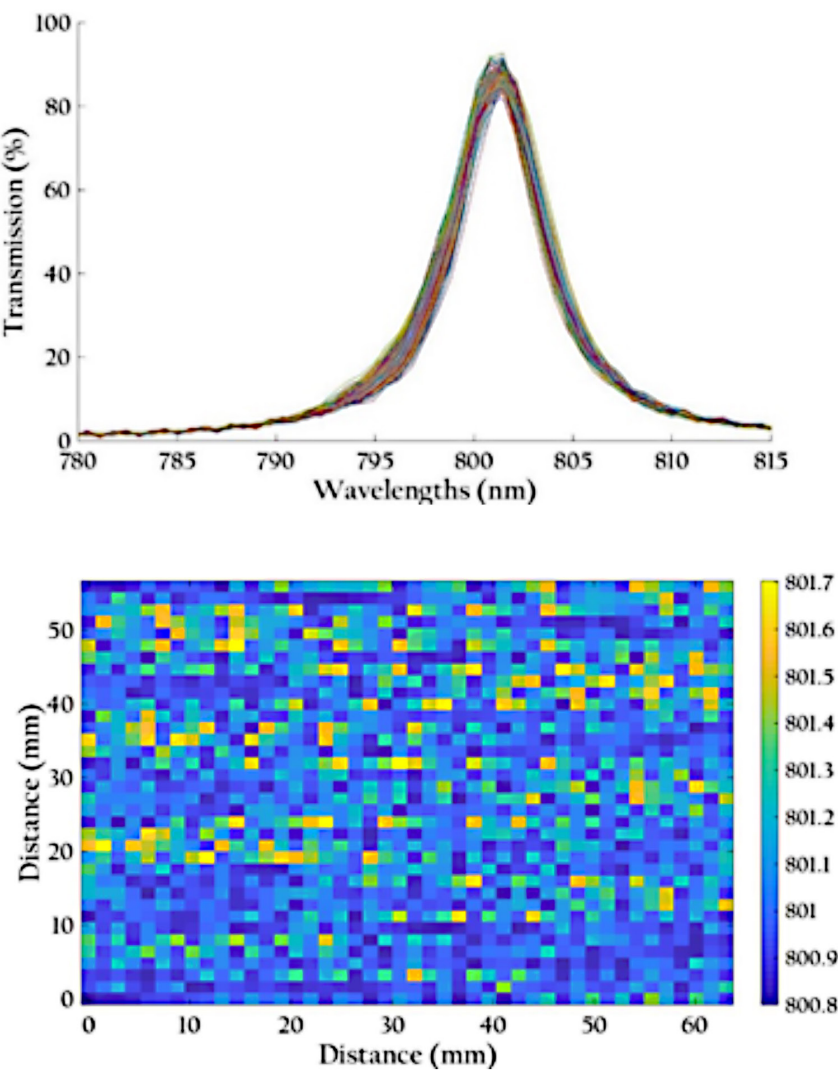

Fig. 7 - Spectral dependence of the transmission measured on each $1 \mathrm{~mm}^{2}$ spot over a $65 \times 60 \mathrm{~mm}^{2}$ aperture after correction of the filter (top) and mapping of the central wavelength of the filter after correction (bottom).

change of maximum transmission or bandpass shape confirming that the proposed is valid for more complex filters

\section{CONCLUSIONS}

We have shown in this letter the possibility to correct, after deposition, the local non-uniformity of narrow bandpass filters.

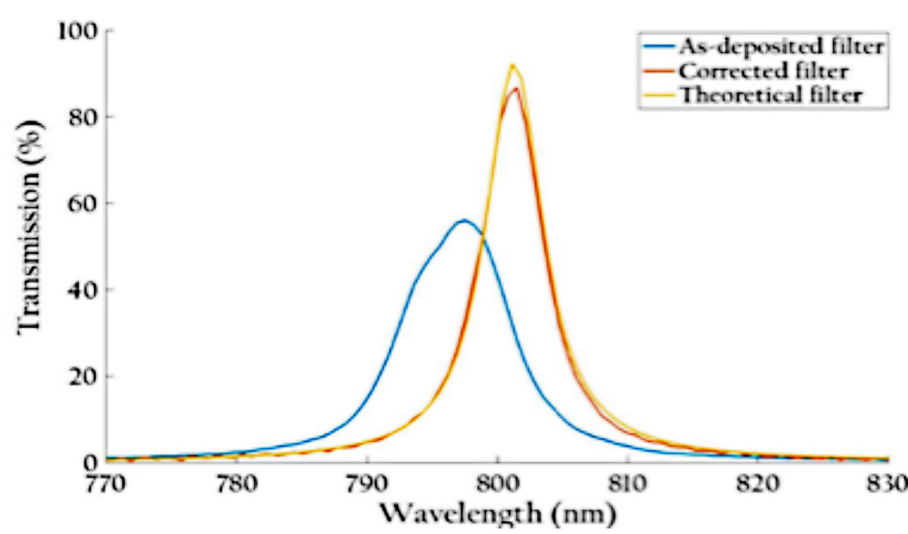

Fig. 8 - Behavior of the filter using a large beam $(50 \mathrm{~mm}$ diameter beam) 


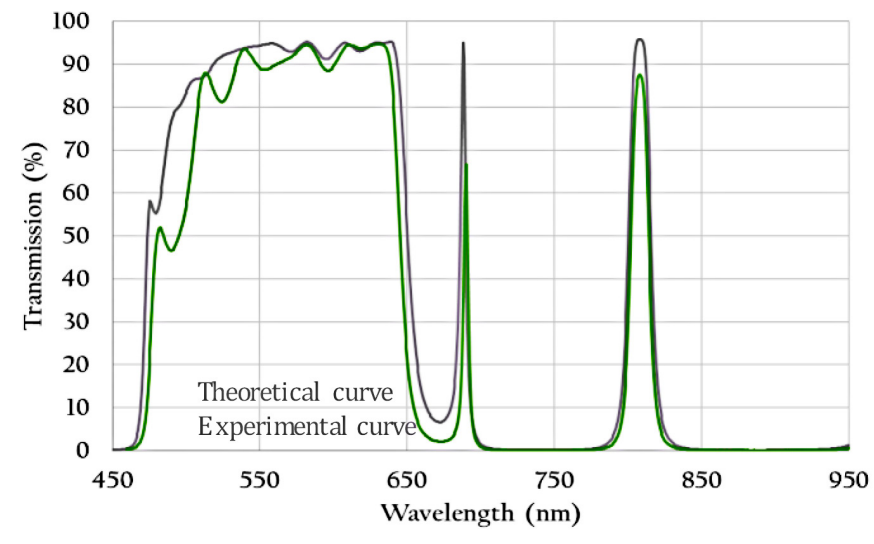

Fig. 9 - Spectral dependence of the transmission of a two-cavity Fabry Perot filter. Theoretical filter: grey curve, experimental filter: green curve.

This correction relies on the use of photosensitive $\mathrm{As}_{2} \mathrm{~S}_{3}$ layers that are used as cavities in Fabry Perot structures. Using a relative refractive index change equal to the relative thickness change (due to non-uniformity issues) and with opposite sign, it is possible to generate highly uniform large aperture bandpass filters. We have shown a method that automatically corrects for the non-uniformity of a filter. Example of a $5 \mathrm{~nm}$ FWHM filter centered at $801 \mathrm{~nm}$ with deviation of the central wavelength less than $0.1 \%$ over $65 \times 60 \mathrm{~mm}^{2}$ squared aperture has been demonstrated. Application of the proposed approach on two-cavity Fabry-Perot filters has also been demonstrated. These results pave a way to the fabrication of large aperture, highly uniform, filters with more complex structures.

\section{ACKNOWLEDGEMENTS}

This work was partially funded by Ministry for Armed Forces (DGA) and Aix-Marseille Université.

\section{REFERENCES}

1. Michel Lequime, Remy Parmentier, Fabien Lemarchand, and Claude Amra, "Toward tunable thin-film filters for wavelength division multiplexing applications," Appl. Opt. 41, 3277-3284 (2002)

2. T. Begou, H. Krol, D. Stojcevski, F. Lemarchand, M. Lequime, C. Grezes-Besset and J. Lumeau, "Complex optical interference filters with stress compensation for space applications”, CEAS Space J 9(4), 441-449 (2017).

3. M. Lequime and J. Lumeau, "Laser trimming of thin-film filters", Advances in Optical Thin-Films (Jena, Germany), invited talk, paper 5963-08, September 2005

4. W.D. Shen, M. Cathelinaud, M. Lequime, V. Nazabal, and X. Liu, "Photosensitive post tuning of chalcogenide Te20As30Se50 narrow bandpass filters," Opt. Commun., 281, 3726-3731 (2008)

5. A. Bourgade et J. Lumeau, "Large aperture, highly uniform narrow bandpass Fabry-Perot filter using photosensitive $\mathrm{As}_{2} \mathrm{~S}_{3}$ thin films ", Opt. Lett., OL, vol. 44, $n^{\circ}$ 2, p. 351-354, January. 2019.

6. H. Hisakuni and K. Tanaka, "Laser-induced persistent self-focusing in $\mathrm{As}_{2} \mathrm{~S}_{3}$ glass", Solid State Communications, Vol. 90, No. 8, pp. 483486, 1994

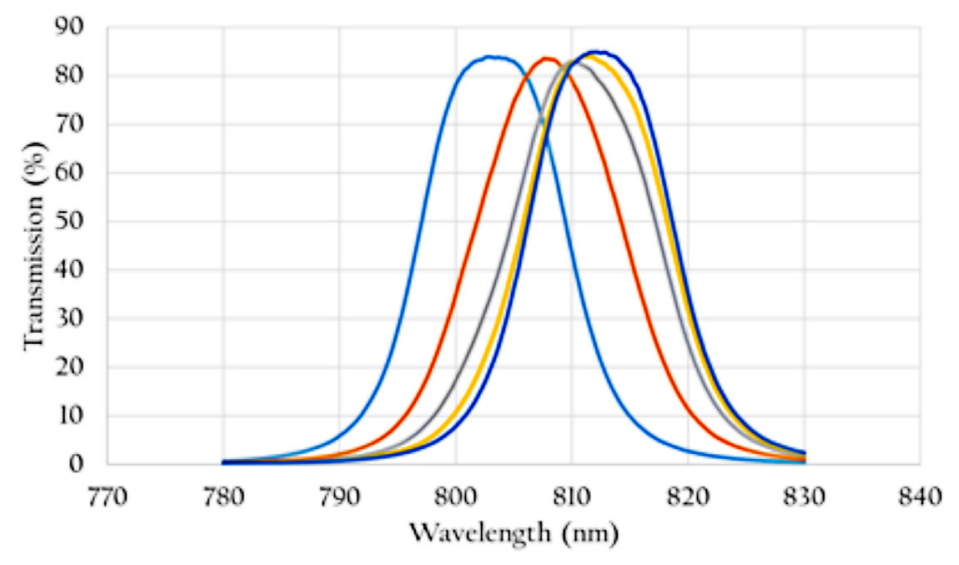

Fig. 10 - Evolution of the bandpass of a two-cavity Fabry-Perot filter for different exposure dosages at $532 \mathrm{~nm}$.

7. K. Tanaka, N. Toyosawa, and H. Hisakuni "Photoinduced Bragg gratings in $\mathrm{As}_{2} \mathrm{~S}_{3}$ optical fibers", Optics Letters, Vol. 20, No. 19, October 1 , 1995

8. C. Meneghini and A.Villeneuve, " $\mathrm{As}_{2} \mathrm{~S}_{3}$ photosensitivity by two-photon absorption: holographic gratings and self-written channel waveguides", J. Opt. Soc. Am. B, Vol. 15, No. 12, December 1998.

9. J. Hu, M. Torregiani, F. Morichetti, N. Carlie, A. Agarwal,et al., "Resonant cavity-enhanced photosensitivity in $\mathrm{As}_{2} \mathrm{~S}_{3}$ chalcogenide glass at $1550 \mathrm{~nm}$ telecommunication wavelength", Optics Letters, Vol. 35, No. 6 , March 15, 2010

10. R. Vallée, S. Frédérick, K. Asatryan, M. Fischer, T. Galstian, "Real-time observation of Bragg grating formation in $\mathrm{As}_{2} \mathrm{~S}_{3}$ chalcogenide ridge waveguides", Optics Communications, Vol 230, No 4-6, pp.301-307, Feb. 2004

11. S. Ramachandran, S.G. Bishop, J.P. Guo, et D.J. Brady. « Fabrication of holographic gratings in $\mathrm{As}_{2} \mathrm{~S}_{3}$ glass by photoexpansion and photodarkening ». IEEE Photonics Technology Letters 8, $\mathrm{n}^{\circ} 8$ (august 1996): 1041-43

12. H. Hisakuni and K. Tanaka, "Optical fabrication of microlenses in chalcogenide glasses," Opt. Lett. 20, 958-960, 1995

13. Gao, L., F. Lemarchand, \& M. Lequime. "Refractive index determination of $\mathrm{SiO}_{2}$ layer in the UV/Vis/NIR range: spectrophotometric reverse engineering on single and bi-layer designs." Journal of the European Optical Society - Rapid publications [Online], 8 (2013)

14. W.D. Shen, M. Cathelinaud, M. Lequime, F. Charpentier, and V. Nazabal, "Light trimming of a narrow bandpass filter based on a photosensitive chalcogenide spacer," Opt. Express, 16, 373-383 (2008)

15. A. Bourgade, F. Lemarquis, T. Begou, A. Moreau and J. Lumeau, "Adjustable three-cavity narrow bandpass filters" To be submitted to Optics Letters (2019)

\section{FOR FURTHER INFORMATION}

Contact Julien Lumeau, Institut Fresnel, head of the Thin-Film research group, Tel. +33 6722890 61, e-mail: julien.lumeau@ fresnel.fr. 
About the Authors:

\section{A. Bourgade, F. Lemarquis, A. Moreau, T. Begou and J. Lumeau}

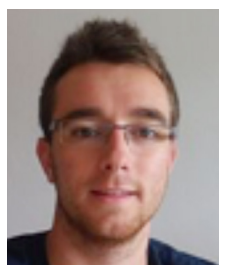

A. Bourgade graduated with an engineering degree from Ecole Centrale Marseille in 2016 and a Ph.D. from Aix Marseille Université in 2019. His thesis aimed at developing chalcogenide-based multilayer structures in order to produce spatially structured optical elements.

F. Lemarquis is Assistant Professor at Ecole Centrale Marseille. He obtained a Ph.D. thesis in 1995. Since then he has been working at Institut Fresnel on the design of thin-film filters for various applications including space projects.

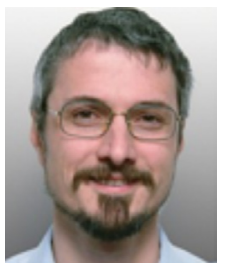

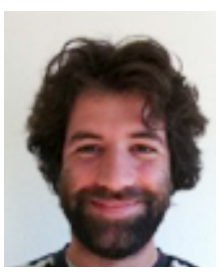

T. Begou obtained a Ph.D. from the Institut des Matériaux Jean Rouxel de Nantes in 2008. He has worked one year as a Posdoctoral researcher at Old Dominion University (Norfolk, Virginia - USA) and then joined Institut Fresnel as an Assistant Professor for Aix Marseille Université. His main expertise includes the fabrication of thin-film filters using plasma assisted reactive magnetron sputtering.

J. Lumeau obtained a Ph.D. from Aix Marseille Université in 2004. He has worked for 8 years as a research scientist at University of Central Florida and OptiGrate company on the development of volume Bragg gratings and then joined Institut Fresnel, in 2012, as a CNRS research scientist. He is the head of the thin film

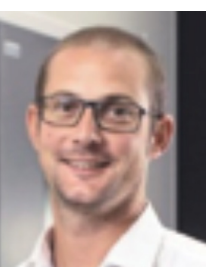
research group and works on the development of complex and structured thin-film components. 